\title{
NON-THERMAL PARTICLES NEAR OB ASSOCIATIONS
}

\author{
ANDREI. M. BYKOV \\ A.F. Ioffe Institute of Physics and Technology \\ 194021, St. Petersburg, Russia
}

\begin{abstract}
The observational tests to check the concept of nonthermal particle generation in the vicinity of $\mathrm{OB}$ associations embedded in a superbubble (SB) are discussed. Acceleration of nonthermal charged particles is expected to be very efficient due to powerful energy release by supernovae and colliding stellar winds. The distinctive features of the SB's emission spectra due to nonthermal particle interaction with gas, dust and electromagnetic fields are summarized.
\end{abstract}

\section{Introduction}

A study of superbubbles and supershells created by stellar winds and multiple supernova explosions in an OB association attracts great attention (see e.g. Heiles (1990), Rand \& van der Hulst, (1993), Yang \& Skillman, (1993)). The possibilities of acceleration of nonthermal particles in the vicinity of an OB-association have been considered in detail by Bykov (1992), Bykov \& Fleishman (1993). The model of particle acceleration has been proposed where the interaction of charged particles with an ensemble of shocks and violent large scale plasma motions inside the bubble was included. The nonthermal charged particle acceleration is expected to be very efficient especially after the explosions of a few supernova in the association. The particle acceleration time is not exceed of $3 \times 10^{5} \mathrm{yr}$ for typical superbubble conditions. Some tens of percent of the kinetic energy released by early type stars and supernovae can be converted to the nonthermal particles. The spectra of accelerated particles are very hard at the energy range up to a few tens of MeV per nucleon $F(p) p^{2} d p \sim N_{n t h} p^{-1} d p$ and then become steeper (here $F(p)$ - is the distribution function of energetic particles in momentum space). 


\section{Nonthermal emission from $O B$ associations}

Here we present the list of expected observable consequences of the interaction of nonthermal particles with magnetic and UV radiation fields inside the SB and with gas and dust of surrounding supershell.

1) Synchrotron radio emission with flat spectrum up to a few hundreds $\mathrm{MHz}$ or $\mathrm{GHz}$ and of steeper radio spectrum (with spectral index of $\sim 0.5-$ 0.8 ) below. Some low degree of polarization is expected from that sources.

2) Hard continuum gamma ray emission due to nonthermal proton and electron interaction with HI shell (see Bykov \& Fleishman, 1993).

3) Gamma - line emission $(0.4 \mathrm{MeV}-10 \mathrm{MeV})$. Gamma ray in the $3-7 \mathrm{MeV}$ range has been detected recently by COMPTEL telescope from Orion complex (Bloemen et al,(1994) and could be identified with deexcitation lines from nonthermal nuclei which accelerated within bubble and interacted with dense clouds just in vicinity of SB (Bykov \& Bloemen, 1994).

4) Optical emission of HeII due to recombination of doubly ionized helium and from the process $H^{+}+H e \rightarrow H^{+}+H e^{+^{*}}+e$. Optical line of HeII $\lambda 4686 \AA$ will be most prominent as it is in the case of some HII regions observed by Garnett et al (1991).

5) Some distinctive spectral features of the IR emission may appear due to stochastic heating of small grains within a dense shells (or nearby clouds) by nonthermal protons and nuclei with energies up to hundreds MeV. Bozhokin \& Bykov, 1995, (in preparation) calculated IR spectra due to heating of the carbon dust grains population of temperature $10 \mathrm{~K}$ and $25 \mathrm{~K}$ with MRN size distribution by nonthermal protons. We expect that the effect of dust heating by nonthermal particles is important for rather old SB without bright stars and with numerous supernova events located in the outer part of the Milky Way or in the other galaxies.

This work was supported in part by the International Science Foundation, grant NU3000 and by Russian Foundation for Fundamental Research.

\section{References}

Bloemen, H. et al., (1994). Astron. Astrophys. 281, L5

Bozhokin S.V., Bykov A.M. (1994), Astron. Lett. 20, 503

Bykov A.M. (1992). In: Evolution of interstellar matter and dynamics of Galaxy eds. J.Palous, W.B.Burton, P.O.Lindblad, Cambridge, p.116

Bykov A., Bloemen H. (1994), Astron. Astrophys. 283, L1

Bykov A.M., Fleishman G.D. (1993), Astron. Astrophys. 280, L27

Chu, Y.-H., Mac Low, M.M. (1990). Astrophys. J. 365, 510

Garnett D.R., Kennicutt R.C., Chu Y.-H., Skillman E.D. (1991), Astrophys. J. 373, 458

Heiles, C. (1990). Astrophys. J. 354, 483

Rand, R.J., van der Hulst, J.M. (1993), Astron. J. 105

Yang, H., Skillman, E.D. (1993), Astron. J. 106, 1448 